\title{
Prevenção de Ansiedade Infantil a partir do Método Friends
}

\author{
Letícia Guancino ${ }^{1}$ \\ Caroline Guisantes de Salvo Toni ${ }^{1}$ \\ Ana Priscila Batista ${ }^{1}$ \\ ${ }^{1}$ Universidade Estadual do Centro-Oeste, Irati, Paraná, Brasil
}

\begin{abstract}
Resumo
Os Transtornos de Ansiedade têm se desenvolvido com mais frequência em crianças em idade escolar, prejudicando sua saúde, relações sociais e desenvolvimento global. Atualmente, tem-se buscado a prevenção desses transtornos. O Método FRIENDS, programa que visa prevenir ansiedade e depressão por meio do desenvolvimento de habilidades socioemocionais, resiliência e comportamentos para uma vida saudável, é uma possibilidade de intervenção. Nesse contexto, esta pesquisa objetivou verificar se a sintomatologia de ansiedade de crianças diminui após a participação destas no Método FRIENDS. Participaram do estudo 19 crianças, com idades entre 5 e 7 anos, de ambos os sexos. Foi utilizado como instrumento de avaliação o Spence Children's Anxiety Scale, respondido pelos pais em pré-teste, pós-teste e follow up. Os dados foram comparados a partir de estatística descritiva e inferencial. Os resultados apontam para a diminuição do total de sintomas de ansiedade nas crianças que participaram do método logo após e no seguimento de dois meses.
\end{abstract}

Palavras-chave: prevenção primária, promoção de saúde, distúrbios da ansiedade, infância, programas de saúde mental

Prevention of Child Anxiety based on the Friends Method

\begin{abstract}
Anxiety disorders have developed more frequently in school-age children, harming their health, social relationships, and overall development. Currently, the prevention of these disorders has been sought. Thus, the FRIENDS Method, a program aimed at preventing anxiety and depression through the development of social-emotional skills, resilience, and behaviors for a healthy life, is a possibility of intervention. In this context, this research aimed to verify whether children's symptoms of anxiety diminish after participation in the FRIENDS Method intervention. A total of 19 children, aged from 5 to 7 years, of both sexes, participated in the study. The Spence Children's Anxiety Scale was used as an assessment tool, answered by the children's parents in the pre-test, post-test, and follow-up. Data were compared using descriptive and inferential statistics. Research findings showed better management of anxiety symptoms by children after participating in the group.

Keywords: primary prevention; health promotion; anxiety disorders; childhood; mental health programs
\end{abstract}

\section{Prevención de Ansiedad Infantil a Partir del Método Friends}

\begin{abstract}
Resumen
Los trastornos de ansiedad se han desarrollado con más frecuencia en ninõs en edad escolar, perjudicando su salud, relaciones sociales y desarrollo global. Actualmente, se ha tratado de prevenir estos trastornos. El Método FRIENDS, es un programa que tiene como objetivo prevenir ansiedad y depresión, por medio del desarrollo de habilidades socioecónomicas, resiliencia y comportamientos para una vida saludable, y también por una posibilidad de intervención. En este contexto, esta investigación tuvo como objetivo verificar si la sintomatología de ansiedad de los niños disminuyó después de la participación de ellos en el Método FRIENDS. Participaran del estudio 19 niños, con edad entre 5 e 7 años, de ambos sexos. Fue utilizado como instrumento de evaluación el Spence Children's Anxiety Scale (SCAS), contestado por los padres en pre-test, post-test y Follow-up. Los datos fueron comparados a partir de una estadística descriptiva e inferencial. Los resultados señalan la diminuición de total de síntomas de ansiedad en los niños que participaron del Método después de dos meses de seguimiento del mismo.

Palabras clave: prevención primaria; promoción de la salud; disturbios de ansiedad; infancia; programas de salud mental
\end{abstract}

\section{Introdução}

Transtornos mentais são cada vez mais conhecidos e frequentes na população mundial, atingindo desde crianças a idosos (Organização Mundial de Saúde [OMS], 2012). Em se tratando de crianças, uma revisão sistemática de Fernandes, Carvalho, Izbicki e Melo (2014) aponta que, em média, 8\% dessa população em idade escolar apresenta sintomas relevantes ou sofre de algum Transtorno de Ansiedade (TA). Vianna, Campos e Fernandez (2009) citam estudos que mostram que a incidência dos transtornos de ansiedade prejudiciais em crianças e adolescente brasileiros chega a 3,40\% e $5,04 \%$, respectivamente. Além de causar danos à saúde mental, esse tipo de transtorno afeta o desempenho escolar, a relação com familiares e amigos e muitas vezes impossibilita o contato social dessas crianças (Asbahr, 2004). 
A ansiedade tem como principais características a autoproteção e a preocupação em relação a eventos que possam trazer perigo à criança ou a seus conhecidos, sendo que a intensidade de manifestação e interferência no dia a dia é o que a faz tornar-se patológica ou não (Asbahr, 2004; Stallard, 2010). A quinta edição do Manual Diagnóstico e Estatístico de Transtornos Mentais (DSM-5; APA, 2014) caracteriza os TA pelo medo e ansiedade excessivos, sendo que o medo é a resposta emocional a situações de ameaça que prepara o sujeito para luta ou fuga. Já a ansiedade é uma reação antecipada de ameaça futura, composta por tensão muscular e alto nível de vigilância, levando a comportamentos de cautela ou esquiva.

Alguns sintomas específicos podem ajudar a identificar a ansiedade na criança. Friedberg e McClure (2004) apontam alguns deles como queixas corporais, tais como dor de barriga, embrulho no estômago, sudorese excessiva e palpitação sem causa clínica, demasiada preocupação e irritabilidade, evitação de eventos ou circunstâncias, medo de falar em público ou de se manifestar, dentre outros.

O DSM-5 (APA, 2014) descreve alguns transtornos de ansiedade que podem acometer crianças, dentre eles: a) Transtorno de Ansiedade de Separação, caracterizado pelo medo excessivo e anormal que a criança sente em separar-se de suas figuras de apego ou de sua casa, de modo que isso prejudique seu desenvolvimento; b) Fobia Social, que se trata do medo excessivo diante de estranhos ou situações de contato social com muitas pessoas em que existe a possibilidade de sentir-se avaliado; c) Pânico, caracterizado por ataques repentinos de medo ou desconforto intenso em que há presença de ansiedade muito forte e medo de morrer, sendo constante o medo de que novos episódios possam ocorrer; d) Agorafobia, caracterizada pelo medo ou evitamento de situações e ambientes muito pequenos, com muitas pessoas ou sem saídas próximas, em que o sujeito possa sentir-se humilhado ou passar vergonha; o medo e a ansiedade geralmente são desproporcionais ao perigo apresentado; e) Transtorno de Ansiedade Generalizada, que se manifesta por meio do sentimento de insegurança, ansiedade e preocupação persistente que a criança apresenta diariamente (Asbahr, 2004; APA, 2014; Stallard, 2002).

As causas dos TA na infância podem estar relacionadas, segundo Weems e Stickle (2005, p. 112), "[...] a uma interação complexa de influências biológicas, cognitivas, comportamentais e sociais [...]", sendo que o peso desses fatores causais pode variar entre as crianças. Desse modo, tornam-se fatores de risco o temperamento vulnerável da criança, o comportamento dos pais (fatores de personalidade e estresse) e fatores ambientais, como baixo nível socioeconômico, abuso infantil, criminalidade, uso de álcool e drogas pelos familiares, entre outros (Izuca \& Barret, 2011).

As consequências da ansiedade na infância envolvem prejuízos escolares, como dificuldades de aprendizagem; prejuízos sociais, como o desenvolvimento de amizades e relações familiares (Stallard, 2012). Também podem ter impacto na trajetória de desenvolvimento geral da criança, por meio do abuso de medicamentos e drogas relacionados ao controle da ansiedade e, em longo prazo, até mesmo a depressão e psicopatologias comórbidas (Stallard, 2002; Vianna, Campos, \& Fernandez, 2009). Quando se trata do prognóstico desses transtornos, estudos (Asbahr, 2004; Pine, Cohen, Gurley, Brook, \& Ma, 1998; Stallard, 2002; Vianna, Campos e Landeira-Fernandes, 2009) apontam que as chances de persistência ao longo da vida da criança são muito grandes, chegando à vida adulta.

Além das dificuldades que a ansiedade pode causar ao longo da vida, gerando sofrimento psíquico acentuado, essa pode acarretar a diminuição de produtividade e incapacitação para o trabalho. Informações apresentadas pela cartilha "Saúde Mental em Dados" (Brasil, 2015) mostram que, em 2014, o governo brasileiro investiu cerca de um bilhão de reais em Centros de Atenção Psicossocial (CAPS), que oferecem serviços de tratamento e acompanhamento para pessoas com transtornos mentais, dentre eles, os transtornos de ansiedade. A Organização Mundial de Saúde (OMS, 2016) estima que até 2030 os gastos mundiais com tratamentos e medicamentos para transtornos mentais cheguem a 147 bilhões de dólares. Sendo assim, existe a necessidade de encontrar alternativas ao tratamento dos transtornos mentais, o que pode contribuir para a diminuição dos números apontados anteriormente, sendo que as maneiras mais indicadas são a prevenção e a promoção de saúde.

A promoção de saúde tem por objetivo fortalecer fatores de proteção como forma de enfrentamento a possíveis determinantes causadores de doenças e transtornos, visando o potencial máximo de saúde das pessoas (Buss, 2009). Por fatores de proteção entendem-se atividades como adoção de políticas públicas, intersetorialidade de ações, criação de ambientes favoráveis à saúde e à aprendizagem de habilidades sociais e emocionais (Brasil, 2015).

Psico-USF, Bragança Paulista, v. 25, n. 3, p. 519-531, jul./set. 2020 
Já a prevenção de saúde, segundo Buss (2009), busca encontrar, controlar e enfraquecer fatores de risco relacionados às doenças com o objetivo de evitar a enfermidade. A prevenção primária, segundo Lohr, Melo, Salvo e Silvares (2013), visa aumentar a saúde e o bem-estar gerais, sem foco em uma doença específica. As estratégias preventivas podem ser caracterizadas quanto ao público que atinge. Chama-se universal quando é direcionada à população em geral, sendo que os benefícios superam os custos e os riscos. A seletiva é direcionada a um grupo específico de risco. Já a indicada se refere a um grupo de pessoas com alto risco de desenvolvimento de doenças (Lohr, Melo, Salvo, \& Silvares, 2013; Murta \& Santos, 2015).

Nesse sentido, a importância de prevenir transtornos e promover saúde mental torna-se primordial quando se trata de transtornos de ansiedade. Juntos, promoção e prevenção ajudam no desenvolvimento da criança como um sujeito capaz de promover resiliência - "[...] conjunto de processos de vida que possibilitam o enfrentamento de situações de sofrimento, transformação pessoal/coletiva/cultural e superação das adversidades [...]" (Yunes, 2014, p. 93-94), e com vasto repertório de habilidades sociais para evitar que possíveis fatores de risco venham a causar prejuízos em sua vida. Murta, Gunther e Guzzo (2015) afirmam que a infância é o momento em que intervenções em saúde mental têm maior importância, pois fortalecem as chances de um desenvolvimento saudável e resiliente diante de situações não favoráveis.

Ao longo dos anos, essa necessidade foi percebida por diversos estudiosos ao redor do mundo (Anticich, Barret, Silverman, Lacherez, \& Gillies, 2013; Iizuka, Barrett, Gillies, Cook, \& Miller, 2014; Kosters et al., 2012). Disso, surgiram programas que unem prevenção e promoção de saúde que são de caráter universal. Eles se mostram vantajosos porque, além de terem longo alcance, possibilitam a redução de diversos problemas sem apontar ou discriminar os participantes (Fernandes, Carvalho, Izbicki, \& Melo, 2014). Programas universais também foram mais eficazes quando comparados a programas seletivos e indicados (Neil \& Christensen, 2009).

Dentre os programas existentes, o FRIENDS (Barret, 1998; Barret, Farrell, Ollendick, \& Dadds, 2006) é um método de abrangência universal criado pela psicóloga Paula Barret, na Austrália. Ancorado em evidências científicas, com base na Terapia Cognitivo-Comportamental (TCC), Psicologia Positiva e fundamentos neurocientíficos, tem por objetivo desenvolver resiliência emocional, promover hábitos de vida saudável e prevenir ansiedade e depressão em seus participantes por meio do ensino de habilidades cognitivas para resolução de problemas, bem como identificação e controle de respostas fisiológicas, reestruturação cognitiva, treinamento de atenção e exposição gradual a situações de medo; para isso, valoriza a participação de familiares, amigos e comunidade ao longo de sua ocorrência (Izuca \& Barret, 2011).

Esses aspectos são trabalhados de diferentes maneiras nas faixas etárias que o programa abrange. Por isso, possui quatro versões: Fun FRIENDS (4 a 7 anos), FRIENDS for Life (7 a 10 anos), My FRIENDS (11 a 17 anos), Adult Resilience (17 em diante) (Barret, 2012).

FRIENDS é um acrônimo em que cada letra representa um objetivo visado pelo método, com o nome traduzido para "AMIGOS" no Brasil. Conforme Izuca e Barret (2011) cada letra de "AMIGOS" se refere aos respectivos objetivos: Atenção aos sentimentos $\left(2^{\circ}\right.$ e $3^{\circ}$ encontros); Momento de relaxar ( $4^{\circ}$ encontro); Ideias que ajudam ( $5^{\circ}$ e $6^{\circ}$ encontros); Gerar soluções ( $7^{\circ}$ e $8^{\circ}$ encontro); Orgulhe-se do seu trabalho $\left(9^{\circ}\right.$ e $10^{\circ}$ encontros); e Sorria e lembre-se de ser corajoso (Barret, 2012). Este último objetivo trata-se de uma habilidade transversal, trabalhada em todos os encontros. Desse modo, o programa é realizado em dez encontros semanais, que contam com o apoio de dois encontros de reforço subsequente ao método (Barret, 2012).

Para o Fun FRIENDS, FRIENDS for Life e My FRIENDS (Barrett, 1998, 2012), acrescentam-se dois encontros realizados com os pais ou comunidade para fornecer informações sobre como dar apoio para as crianças e adolescentes que participam do programa. Dessa forma, eles se tornam capazes de entender melhor as preocupações dos filhos, bem como utilizar uma linguagem semelhante à utilizada nos encontros; envolver os cuidadores no programa proporciona um aumento da probabilidade de que as crianças possam manter as estratégias de resiliência aprendidas, bem como dar ênfase à importância do suporte familiar e dos pares (Barret, 2012). Os cuidadores também participam dos últimos 10 minutos de cada sessão das crianças e adolescentes, para que possam ser informados sobre os conteúdos tratados no dia, e também para que os facilitadores possam modelar o comportamento destes (Figueredo, no prelo). Evidências mostram que quando os cuidadores participam ativamente do programa, por meio das sessões para comunidade, a eficácia do método na diminuição da sintomatologia de ansiedade das crianças é maior (Barret, 2012; Figueredo, no prelo). 
O método FRIENDS (Barrett, 1998, 2012) vem sendo aplicado e pesquisado em diversos países, com estudos de validade positivos publicados na Espanha (Tortella-Feliu, Servera, Balle, \& Fullana, 2004), Austrália (Anticich et. al. 2013, Iizuka et al., 2014), Inglaterra (Stallard et. al. 2005), Holanda (Kosters et al, 2012), Suécia (Ahlen, Breitholtz, Barret, \& Gallegos, 2012), Brasil (Pavoski, Toni, Batista, \& Ignachewski, 2018), Escócia (Liddle \& Macmillan, 2010) entre outros, apresentando ótimos resultados em diminuição de sintomas de ansiedade e depressão e aumento de repertório de habilidades socioemocionais em suas diversas versões (NREPP, 2012).

Um exemplo de país que tem utilizado o método FRIENDS (Barrett, 1998, 2012) é a Austrália, local em que ele foi criado e no qual hoje está presente em forma de política pública. $\mathrm{O}$ primeiro estudo realizado para avaliação do método, em 1998, teve como objetivo verificar sua eficácia na diminuição de sintomatologia de ansiedade na infância, contando com a participação de 60 crianças. Os resultados apontaram que as crianças que participaram do método, quando comparadas ao grupo-controle, tiveram uma redução significativa dos sintomas de ansiedade nas avaliações pós-teste e follow up, indicando a eficácia do método (Barret, 1998; Figueredo, no prelo).

Entre diversos estudos com o Fun Friends pode-se destacar o estudo de Anticich, Barret, Silverman, Lacherez e Gillies (2013). Neste, participaram 488 crianças, com idades entre 4 e 7 anos, matriculados regularmente em escolas. Os participantes foram classificados a partir das respostas de seus pais e de seus professores a diferentes instrumentos que avaliavam sintomatologia de ansiedade em grupo de risco e grupo com baixa sintomatologia de ansiedade. Os resultados demonstraram redução de sintomas ansiogênicos nos dois grupos que se mantiveram na avaliação de follow up, 12 meses após o término do método, ou seja, o método mostrou-se evidências de eficácia tanto na prevenção universal, quanto indicada. Ainda, destaca-se que nesse estudo observou-se mudanças no comportamento dos cuidadores dos participantes após a participação das crianças no método. Os cuidadores diminuíram seus níveis de estresse, bem como apresentaram uma visão mais positiva dos comportamentos da criança. Esses dados sugerem que após a participação no método FRIENDS, fez-se possível maior flexibilidade de comportamentos parentais, como controle e proteção excessiva, bem como diminuição de frustração e maior compreensão da ansiedade de seus filhos.
Estudos iniciais com o método no Brasil demonstraram diminuição dos sintomas de ansiedade e depressão, bem como melhora nos comportamentos das crianças participantes, sendo que a avaliação em follow up de dois meses demonstrou a manutenção dos ganhos (Pavoski et al., 2018). Porém, no Brasil ainda existem poucos estudos de eficácia do programa, que foi traduzido para o português-brasileiro apenas em 2012 (Zeggio \& Araújo, 2015). Desse modo, pesquisar e buscar evidências de validade para o programa para a população brasileira é o primeiro passo para que ele possa ser implementado por meio de política pública em diferentes contextos como na educação, saúde e assistência social.

É de extrema importância que esse tipo de programa seja de acesso da população geral, pois implica na prevenção de transtornos como ansiedade e depressão e promove saúde mental como um todo. Tendo isso em vista, esta pesquisa teve como objetivo verificar se a sintomatologia de ansiedade de crianças diminui após a participação destas no método FRIENDS (Barrett, 1998, 2012), bem como avaliar se tais resultados se mantêm após um seguimento de dois meses, com vistas a verificar sua validação no contexto de uma cidade no interior do Paraná.

\section{Método}

\section{Participantes}

Participaram deste estudo 19 crianças, com idade entre cinco e sete anos (média de idade de 6,55 anos), de ambos os sexos (13 meninos e 6 meninas), bem como seus pais e cuidadores. As crianças participaram em dois grupos distintos de aplicação do programa FRIENDS em uma clínica-escola de Universidade Pública do interior do Paraná ao longo dos anos de 2016 e 2017: Grupo $1(n=10)$, sendo nove estudantes de escolas particulares, Grupo $2(n=9)$ sendo todos estudantes de escolas públicas [Escola Particular $=9$, Escola Pública $=10]$. Apenas dois critérios de inclusão para a composição da amostra foram utilizados: crianças com idade entre 5 a 7 anos e pais/cuidadores com disponibilidade de participar das atividades do programa (10 minutos ao final de cada encontro das crianças e dois encontros de orientação para pais).

A idade média dos pais das crianças foi de 38 anos e das mães 34 anos. Do total de participantes, 18 crianças $(72 \%)$ moravam com pai e mãe, enquanto sete $(28 \%)$ residiam com apenas um dos pais.

Psico-USF, Bragança Paulista, v. 25, n. 3, p. 519-531, jul./set. 2020 


\section{Instrumentos}

$\mathrm{O}$ instrumento utilizado para verificação dos sintomas de ansiedade foi o SCAS - Spence Children's Anxiety Scale (Spence, 1999). Trata-se de uma escala que visa medir os níveis de sintomatologia de ansiedade infantil por meio de respostas fornecidas pelos cuidadores das crianças. É composto por 38 itens que descrevem comportamentos da criança correlatos a sintomas de ansiedade. Os responsáveis respondem de acordo com a frequência com que os comportamentos descritos nos itens ocorrem em uma escala Likert de quatro pontos: nunca, às vezes, muitas vezes, sempre. Os 38 itens se dividem em seis escalas, seis itens abordam questões sobre ansiedade de separação, seis sobre fobia social, seis sobre problemas de obsessão compulsiva, seis sobre pânico, três sobre agorafobia, seis sobre ansiedade generalizada e seis sobre medos de injúria corporal. A somatória absoluta dos itens relativos a cada escala forma escores, sendo que o somatório absoluto de todos os itens dá origem à Escala Total de Sintomas de Ansiedade. Os dados preliminares de validação e normatização do SCAS (Spence, 1999) para a população brasileira mostraram sua validade e a possibilidade de sua utilização nesse contexto (DeSousa et. al., 2014, Graeff-Martins \& Fleitlich-Bilyk, 2016).

\section{Procedimentos}

Este projeto foi aprovado pelo Comitê de Ética da Universidade Estadual do Centro-Oeste pelo $\mathrm{N}^{\circ}$ : 65208116.1.0000.0106. Em seguida à sua aprovação, realizou-se a seleção dos participantes.

\section{Recrutamento}

Os dados da presente pesquisa são provenientes de dois grupos de crianças que participaram do Amigos Divertidos em uma Clínica-Escola de uma Universidade Pública do interior do Paraná nos anos de 2016 e 2017. Para que o projeto fosse executado, inicialmente as pesquisadoras e estagiárias participaram de uma formação, fornecida por treinadora habilitada internacionalmente pelo método FRIENDS, para poder atuar como facilitadoras do programa.

Para compor os grupos, foram utilizadas duas estratégias: a) contato com a Clínica-Escola de Psicologia para identificar crianças na lista de espera para psicoterapia que atendessem ao critério de idade da pesquisa (cinco a sete anos), independente da queixa informada no ato da inscrição para atendimento. Apenas uma criança preenchia critério de idade da pesquisa, e ela e seus pais aceitaram participar do grupo $(n=1)$; b) contato com escolas do município e convite para os pais/cuidadores via agenda escolar. Nesse convite estavam descritas informações sobre o Programa e como inscrever-se de forma gratuita ligando para a Clínica-Escola de Psicologia. Todas as crianças inscritas dessa forma $(n=24)$ participaram do estudo, sendo que se seguiu a ordem de inscrição para construção dos grupos. Destaca-se que não havia critério de nível de sintomatologia de ansiedade na criança para que ela pudesse ser incluída na pesquisa, tendo em vista que o método FRIENDS (Barrett, 1998, 2012) busca a prevenção universal da ansiedade.

\section{Coleta de Dados}

Antes de se iniciarem as intervenções, os pais/ cuidadores foram convidados a uma entrevista inicial na qual foi apresentado o método Fun FRIENDS e a pesquisa, pelas facilitadoras. Aqueles que aceitaram participar assinaram o Termo de Consentimento Livre e Esclarecido (TCLE) e responderam ao SCAS (Spencer, 1999) (pré-teste) de forma individual, com a presença de uma das pesquisadoras.

Para a realização do programa, foi utilizada a versão adaptada do método FRIENDS para o Brasil, a qual se encontra disponível no Manual Amigos Divertidos (Barrett, 2012). Os participantes foram divididos em dois grupos, tendo em vista o espaço físico disponível para realização do grupo. O primeiro contou com dez crianças e foi realizado em 2016. Já o segundo grupo teve a participação de nove crianças, sendo realizado em 2017. Ambos os grupos passaram pela intervenção e seguiram a estrutura do método Fun FRIENDS (Barret, 2012), com dez encontros de frequência semanal com temáticas pré-estabelecidas e resumidas na Tabela 1.

Após a finalização do décimo encontro, foi realizado novo com os responsáveis para preenchimento do SCAS (Spencer, 1999) em pós-teste. Os dois encontros de reforço do método aconteceram 30 e 60 dias respectivamente após sua finalização. Por ocasião do segundo encontro de reforço, foi realizada a avaliação de follow up, e três cuidadores não compareceram. Tanto a avaliação de pós-teste, quanto a de follow up ocorreu de forma coletiva e com a presença de uma facilitadora para que pudesse sanar eventuais dúvidas dos pais. $\mathrm{O}$ delineamento do presente estudo caracteriza-se como quase-experimental.

\section{Análise de Dados}

Os dados do instrumento SCAS (Spencer, 1999) foram sistematizados e analisados com auxílio do 
Tabela 1.

Objetivos e Estratégias nas Sessões do Método Fun Friends

\begin{tabular}{|c|c|c|}
\hline Sessão & Objetivos do encontro & Estratégias \\
\hline 1 e 2 & $\begin{array}{l}\text { Atenção aos sentimentos: apresentação dos participantes; } \\
\text { Identificar, compreender e expressar sentimentos, bem } \\
\text { como seus sinais fisiológicos. }\end{array}$ & $\begin{array}{l}\text { Bola com adesivos para apresentação; } \\
\text { dado das emoções. }\end{array}$ \\
\hline 3 & $\begin{array}{l}\text { Momento de relaxar: entender a importância do } \\
\text { relaxamento para lidar com preocupações e emoções. }\end{array}$ & $\begin{array}{l}\text { Estratégias de relaxamento: } \\
\text { respiração milk-shake; massagem } \\
\text { pizza. }\end{array}$ \\
\hline 4 e 5 & $\begin{array}{l}\text { Ideias que ajudam: compreender o conceito de diálogo } \\
\text { interno (self-talk) através dos pensamentos verdes, amarelos } \\
\text { e vermelhos; transformar pensamentos vermelhos em } \\
\text { verdes. }\end{array}$ & $\begin{array}{l}\text { Brincadeira do semáforo: pare, pense, } \\
\text { siga. }\end{array}$ \\
\hline 6 e 7 & $\begin{array}{l}\text { Gerar soluções: para resolver conflitos, é importante } \\
\text { explorar soluções, criar um plano passo a passo para } \\
\text { solucioná-los e ter uma pessoa modelo. }\end{array}$ & $\begin{array}{l}\text { Separar metas para atingir um grande } \\
\text { objetivo; montar quebra cabeça. }\end{array}$ \\
\hline 8 e 9 & $\begin{array}{l}\text { Orgulhe-se do seu trabalho: aprender a recompensar-se } \\
\text { com coisas não materiais. }\end{array}$ & $\begin{array}{l}\text { Escolher uma brincadeira; organizar } \\
\text { uma festa. }\end{array}$ \\
\hline 10 & $\begin{array}{l}\text { Sorria e lembre-se de ser corajoso: encoraja os participantes } \\
\text { a praticar as habilidades aprendidas nos encontros. }\end{array}$ & $\begin{array}{l}\text { Diário da gratidão; livro de atividades } \\
\text { Fun Friends. }\end{array}$ \\
\hline
\end{tabular}

Nota. Tabela baseada em Barret, 2012; Izuca e Barret, 2011.

software SPSS 18.0 (Statistical Package for the Social Sciences) utilizando estatística descritiva e inferencial. Para a comparação dos dados em pré e pós-teste, pós-teste e follow up, e pré-teste e follow up, foi empregado o teste estatístico não paramétrico de Wilcoxon (Wilcoxon, 1945). O teste de Wilcoxon é utilizado para comparar amostras pareadas a partir da comparação de dois momentos de avaliação de um mesmo indivíduo. Por meio dessa comparação, tem-se o escore $Z$ e $p$. O escore $p$ quando $\leq 0,05$ representa que as diferenças foram estatisticamente significativas. Foi também verificado o tamanho de efeito das diferenças observadas a partir da fórmula de $d$ de Cohen. De acordo com Espírito-Santo $\&$ Daniel (2015) considera-se os valores de $d$ até 0,19 como insignificantes; 0,20 a $0,49=$ pequeno; 0,50 a 0,79 $=$ médio; $\geq 0,80=$ grande .

\section{Resultados}

Os dados referentes à sintomatologia de ansiedade das 19 crianças dos dois grupos foram comparados a partir de estatística inferencial utilizando-se o teste $\mathrm{U}$ de Mann-Whitney, pois os participantes de um grupo eram provenientes de escola pública, e do outro de escola privada. Esse teste busca verificar em uma amostra pequena, na qual a variável numérica não apresenta uma variação normal, se dois grupos não pareados pertencem ou não à mesma população. Os resultados da comparação dos dois grupos mostraram-se iguais no ponto de vista estatístico em todas as escalas do SCAS (ex., total de sintomas de ansiedade $\mathrm{U}=41,5$; $p=0,775)$. A partir da comparação realizada, e tendo em vista a homogeneidade dos dois grupos, foi possível optar no presente trabalho por apresentar os dados dos dois grupos em conjunto.

No primeiro grupo, 10 crianças participaram do pré e pós-teste. Já no follow up, apenas oito crianças. No segundo grupo todas as nove crianças participaram das três etapas de avaliação. Desse modo, 19 crianças contabilizam no pré e pós-teste e 17 no follow up.

Os resultados serão apresentados em dois momentos: na Tabela 2 as médias e desvio padrão das escalas do instrumento em cada etapa da avaliação; na Tabela 3 os resultados da análise inferencial a partir do teste de Wilcoxon e teste de tamanho de efeito de Cohen.

Na Tabela 2 estão dispostas as escalas avaliadas no SCAS, bem como as médias e desvio padrão em cada fase da avaliação. Pode-se observar que as médias de 
três escalas - ansiedade de separação, ansiedade generalizada e total de sintomas - tendem a diminuir da primeira para a terceira fase. Já as médias das escalas agorafobia, medo de injúria física e obsessão compulsiva diminuem do pré-teste para o pós-teste, mas sofrem um leve aumento na fase de follow up em relação ao pós-teste. Em todas essas escalas, as médias do follow up diminuíram em relação ao pré-teste. Apenas o item fobia social sofreu aumento da primeira para a segunda fase. Porém, na terceira fase o escore diminuiu, ficando menor que no pré-teste.

A Tabela 3 apresenta os valores da análise inferencial de comparação da amostra nos três momentos de avaliação: pré-teste e pós teste; pós-teste e follow up; pré-teste e follow up.

Partindo da Tabela 3, pode-se verificar que, na comparação do pré-teste com o pós-teste, foram observadas diferenças estatisticamente significativas em três escalas: ansiedade de separação $(Z=-2,033 ; p=0,01)$, obsessão compulsiva ( $\mathrm{Z}=-2,06 ; p=0,03)$, e no total de sintomas $(Z=-2,31 ; p=0,02)$. Quanto ao tamanho do efeito das diferenças observadas, destaca-se que para essas três escalas foram efeitos médios ( $d$ acima de 0,50). Ainda, apesar de não se observarem diferenças estatisticamente significativas nas outras escalas, observou-se uma diferença entre os resultados dos dois momentos de avaliação com efeito pequeno, porém, não insignificante.

Já na comparação pós-teste com follow up, apenas a escala de ansiedade generalizada $(\mathrm{Z}=-2,18 ; p=0,02)$ apresentou diferença estatisticamente significante $\mathrm{e}$ com tamanho de efeito médio. Por fim, ao comparar o pré-teste com o follow up, foram encontradas quatro escalas com diferenças estatisticamente significativas e com tamanho de efeito médio: ansiedade de separação $(\mathrm{Z}=-2,66 ; p=0,008)$, obsessão compulsiva $(\mathrm{Z}=-2,55$;

Tabela 2.

Descrição de Média e Desvio Padrão das Escalas do SCAS nas Três Etapas de Avaliação

\begin{tabular}{lcccccc}
\hline \multirow{2}{*}{\multicolumn{1}{c}{ Escalas }} & \multicolumn{2}{c}{ Pré-teste } & \multicolumn{2}{c}{ Pós-teste } & \multicolumn{2}{c}{ Follow up } \\
\cline { 2 - 7 } & Média & $D P$ & Média & $D P$ & Média & $D P$ \\
\hline Agorafobia & 1,11 & 1,48 & 0,37 & 0,59 & 0,53 & 0,943 \\
Ansiedade de Separação & 6,42 & 3,77 & 4,47 & 2,59 & 3,94 & 2,43 \\
Medo de Injúria Física & 3,74 & 2,66 & 3,16 & 2,26 & 3,53 & 2,45 \\
Fobia Social & 3,42 & 2,54 & 4,32 & 2,21 & 3,12 & 2,52 \\
Obsessão/ Compulsão & 1,79 & 2,09 & 0,79 & 1,13 & 0,82 & 1,07 \\
Ansiedade Generalizada & 5,21 & 2,09 & 4,37 & 1,64 & 3,71 & 2,05 \\
Total de Sintomas & 21,68 & 9,03 & 17,47 & 6,99 & 15,59 & 7,25 \\
\hline
\end{tabular}

Tabela 3.

Comparação dos Escores do SCAS nos Três Momentos de Avaliação do Programa, por meio do Teste de Wilcoxon e Tamanho do Efeito a partir do d de Cohen

\begin{tabular}{lcccccc}
\hline \multicolumn{1}{c}{ Escalas } & Pré-Pós & $d$ & Pós-Follow & $d$ & Pré- Follow & $d$ \\
\hline Agorafobia & $\mathrm{z}=-1,80$ & 0,46 & $\mathrm{z}=-0,31$ & 0,10 & $\mathrm{z}=-0,58$ & 0,20 \\
Ansiedade de Separação & $\mathrm{z}=-2,33^{* *}$ & 0,54 & $\mathrm{z}=-1,16$ & 0,31 & $\mathrm{z}=-2,66^{* *}$ & 0,75 \\
Medo de Injúria Física & $\mathrm{z}=-1,26$ & 0,30 & $\mathrm{z}=-0,44$ & 0,13 & $\mathrm{z}=-0,31$ & 0,10 \\
Fobia Social & $\mathrm{z}=-1,72$ & 0,43 & $\mathrm{z}=-1,87$ & 0,47 & $\mathrm{z}=-0,04$ & 0,05 \\
Obsessão Compulsiva & $\mathrm{z}=-2,06^{*}$ & 0,53 & $\mathrm{z}=-0,182$ & 0,04 & $\mathrm{z}=-2,55^{* *}$ & 0,73 \\
Ansiedade Generalizada & $\mathrm{z}=-1,44$ & 0,34 & $\mathrm{z}=-2,18^{*}$ & 0,55 & $\mathrm{z}=-1,98^{*}$ & 0,50 \\
Total de Sintomas & $\mathrm{z}=-2,31^{*}$ & 0,52 & $\mathrm{z}=-1,51$ & 0,45 & $\mathrm{z}=-2,60^{* *}$ & 0,74 \\
\hline
\end{tabular}

Nota. ${ }^{*} p \leq 0,05 ; * * p \leq 0,01 ; * * * p \leq 0,001$. 
$p=0,01)$, ansiedade generalizada $(Z=-1,98 ; p=0,04) \mathrm{e}$ total de sintomas $(\mathrm{Z}=-2,60 ; p=0,009)$.

\section{Discussão}

A análise de eficácia do método FRIENDS (Barrett, 1998, 2012) foi realizada a partir da comparação de sintomas de ansiedade antes da participação do grupo, após e em follow up de dois meses, por meio do instrumento SCAS (Spencer, 1999). Da sintomatologia de ansiedade avaliada pelo SCAS, dois conjuntos de sintomas têm habilidades para seu manejo desenvolvidas diretamente pelo método FRIENDS (Barrett, 1998, 2012): fobia social e ansiedade generalizada. Também o total de sintomas de ansiedade, que deriva de todas as escalas dos SCAS (Spencer, 1999), pode ter seus resultados alterados pela participação da criança no método FRIENDS (Izuka \& Barret, 2011). Já os sintomas avaliados pelas outras escalas do SCAS (Spencer, 1999) não são especificamente trabalhados nas atividades do método FRIENDS (Barrett, 1998, 2012), podendo se beneficiar de forma indireta pelo desenvolvimento de repertórios comportamentais construídos.

A partir da visão dos pais acerca do comportamento de seus filhos, é possível verificar que o método proporcionou resultados significativos em relação à diminuição de ansiedade, quando comparadas às três etapas de avaliação no que tange à escala total de sintomas. Ou seja, quando comparados os resultados pós-intervenção com os de linha de base, observa-se que esses diminuíram e se mantiveram em decréscimo no follow up. A escala total de sintomas é caracterizada pela soma de todas as escalas do SCAS (Spencer, 1999), de modo que sua diminuição se dá em função da baixa do conjunto de sintomas avaliados pelo instrumento.

A eficácia do método FRIENDS (Barrett, 1998, 2012), no que tange à redução da sintomatologia de ansiedade infantil, vem sendo observada em estudos de diversos países como Austrália (Anticich et. al. 2013), Inglaterra (Stallard et al., 2005) e Espanha (Tortella-Feliu et. al. 2004). Os dados do presente estudo vão ao encontro destes e de outros estudos realizados com o método FRIENDS (Barrett, 1998, 2012), que apontam para a prevenção e redução dos sintomas de ansiedade (Ahlen et al., 2012), bem como redução de evitação cognitiva e de situações estressantes em crianças de 4 a 7 anos (Lock \& Barret, 2003). Destaca-se que a diminuição da sintomatologia total de ansiedade após a participação no método, bem como avaliação pré-teste, pós-teste e follow up são algumas das similaridades observadas entre o presente estudo e os estudos internacionais. Tendo em vista que os artigos internacionais supracitados utilizaram metodologias experimentais com grupo controle e observaram resultados semelhantes ao do presente estudo, supõe-se que os resultados aqui observados apontam para um possível efeito de eficácia do método para a população estudada. Salienta-se ainda que a observação do tamanho de efeito moderado dos resultados, também sugerem que as mudanças verificadas na sintomatologia de ansiedade dos participantes não ocorreram ao acaso na população, visto que clinicamente uma magnitude de efeito moderado é um índice clinicamente relevante (Espírito-Santo \& Daniel, 2015).

Em um estudo de revisão abordando programas que vêm sendo desenvolvidos para prevenção universal da ansiedade na infância, Fernandes et. al. (2014) assinalam que o método FRIENDS (Barrett, 1998, 2012) é o programa de prevenção universal de ansiedade para crianças e adolescentes mais largamente aplicado e avaliado positivamente no mundo. As atividades do programa que são realizadas nos grupos trabalham habilidades socioemocionais, de resiliência, estratégias de manejo de estresse por meio do reconhecimento de emoções em si e nos outros, manejo das emoções, treino de empatia, relaxamento, resolução de problemas e conflitos, reconhecimento de pensamentos disfuncionais e busca de assistência, entre outros. Essas habilidades possibilitam que a criança desenvolva autorregulação emocional e comportamental (Barret, 2012; Iizuka \& Barrett, 2011; Stallard, 2010; Zeggio \& Araújo, 2015). Desse modo, contribui também para a diminuição dos sintomas de ansiedade e possibilita o enfrentamento de situações estressantes (Lock \& Barret, 2003). Além disso, a aquisição dessas habilidades aumenta fatores de proteção, que evitam o surgimento de problemas de saúde mental, desajustamento e baixo desempenho escolar, contribuindo com o desenvolvimento biopsicossocial e bem-estar geral da criança (Figueredo, no prelo; Stallard, 2010).

A participação dos cuidadores é de suma importância para que o método FRIENDS seja efetivo (Barret, 1998; Lock \& Barret, 2003). Os dois encontros realizados com a família ou comunidade, bem como a presença dos pais ao final de cada sessão possibilitam a generalização dos resultados para o contexto natural da criança. Ao receber orientações e conhecer os conteúdos trabalhados no método, os cuidadores têm sua confiança reforçada e maior conscientização acerca dos 
comportamentos ansiogênicos das crianças, de modo que aprendem estratégias de enfrentamento positivas para ajudar seus filhos a regularem as emoções (Anticich et al., 2013).

Em relação às outras escalas que analisam a sintomatologia de ansiedade, observa-se que os sintomas de ansiedade de separação e ansiedade generalizada sofreram redução significativa quando comparados o pré-teste com o follow up. Segundo Iizuka e Barret (2011), os sintomas de fobia social, ansiedade generalizada e ansiedade de separação apresentam-se num continuum, que abrange desde as fobias específicas até medos gerais e difusos, por isso estão relacionadas. $\mathrm{O}$ método FRIENDS (Barrett, 1998, 2012) oferece subsídios para a diminuição desses sintomas a partir da identificação dos sintomas corporais, cognitivos e emocionais, para então desenvolver o treino de relaxamento e enfrentamento.

Algumas das atividades realizadas que possibilitam a diminuição desses sintomas envolvem o reconhecimento das emoções e sensações corporais, bem como o manejo dessas emoções, que pode ser praticado por meio da respiração diafragmática e relaxamento muscular progressivo, plano passo a passo para exposição gradual e resolução de problemas (Iizuka \& Barrett, 2011; Lock \& Barret, 2003; Stallard, 2010). Essas habilidades influenciam em como a criança vai se comportar diante de situações problemas, como o medo de separar-se dos pais ou de falar em público. Nos encontros, elas eram motivadas a serem corajosas e pensar em como enfrentar seus medos em pequenos passos, por meio da exposição gradual aos estímulos temidos em uma escala hierarquizada do estímulo de menor para o de maior intensidade.

Apesar de se compreender que os sintomas de fobia social, ansiedade de separação e ansiedade generalizada encontram-se em um contínuo, não se observou mudanças estatisticamente significativas nos escores da escala fobia social. Destaca-se, porém, que as médias nessa escala já eram baixas no pré-teste do método, o que pode ter colaborado para não se verificar diferenças significativas do ponto de vista estatístico nos três momentos de avaliação. Observou-se sutis mudanças, como as médias em follow up serem mais baixas em comparação ao pré-teste. Ainda, tendo em vista que os dados aqui utilizados são decorrentes da observação dos pais em relação ao comportamento dos filhos, pode-se supor que os pais não estivessem sensíveis a pequenas mudanças do comportamento dos filhos no que tange suas relações socias, pois como afirma
Gillham, Shatte \& Reivich (2001), citados por Pahl \& Barret (2010), pode levar algum tempo para que os participantes passem por algum evento de risco elevado, de modo que os efeitos preventivos do programa podem demorar a serem notados pelos pais das crianças.

Outra sintomatologia que obteve diminuição estatisticamente significativa nos escores foram os comportamentos de obsessão compulsiva. Apesar desses comportamentos não serem o foco do método FRIENDS, é possível que algumas habilidades gerais desenvolvidas pelo programa possam ter colaborado para tais resultados. Dentre as habilidades que podem ter colaborado para essa redução, além das já citadas, estão as que trabalham reestruturação cognitiva, sendo que um dos meios de atingir esse objetivo é por meio do uso do self-talk, reconhecimento de cognições (Stallard, 2010), que instiga as crianças a observarem seus pensamentos e percebê-los de forma positiva, aumentando o diálogo interno (Izuca \& Barret, 2011).

Os sintomas de agorafobia e medo de injúria física - que se caracteriza pelo medo excessivo de machucar-se ou ser machucado (Barret, 2012), não obtiveram diminuição estatisticamente significativa, porém os dados de média e desvio padrão indicam que os resultados do follow up reduziram em relação ao pré-teste, mesmo este não sendo foco do método FRIENDS (Barrett, 1998, 2012). Hipotetiza-se que habilidades como foco atencional em aspectos positivos, identificação de redes de suporte e identificação e expressão de pensamentos, podem ter contribuído para as diferenças de médias nas diferentes fases da avaliação, porém, sem intensidade e frequências suficientes para uma alteração estatisticamente significativa. Ainda se destaca que em tais escalas os participantes já apresentaram em linha de base (pré-teste) escores bastante baixos, que se mantiveram ao longo do processo.

Após a participação das crianças no método FRIENDS (Barrett, 1998, 2012), verificou-se a diminuição estatisticamente significativa das médias de quatro escalas das seis avaliadas pelo SCAS (Spencer, 1999), o que também ocorreu em outros estudos que utilizaram este instrumento (por ex.: Barret, Moore, \& Sonderegger, 2000; Stallard et al., 2005). Destaca-se que nesses estudos, o total de sintomas de ansiedade teve redução, o que foi indicado pelos autores como critério de eficácia do método. No presente estudo, observou-se, com base na literatura, evidências de eficácia do método FRIENDS (Barrett, 1998, 2012), para manejo de ansiedade em contexto de clínica-escola, para crianças com idade entre 5 e 7 anos. 
Apesar de os resultados indicarem o potencial do método FRIENDS (Barrett, 1998, 2012) para a prevenção e redução dos sintomas de ansiedade, limitações do estudo devem ser consideradas. Destaca-se a ausência de metodologia experimental com grupos controle. A falta de grupo controle impede a afirmação de que os resultados observados no presente estudo ocorreram devido a variável participação no método Friends. Também, a amostra por conveniência, o local do desenvolvimento dos grupos e o número pequeno de participantes são variáveis a serem consideradas e que levam a necessidade de interpretação dos resultados com cautela.

A partir dessas questões, e considerando o caráter quase experimental do presente estudo, destaca-se a necessidade de novas pesquisas utilizando delineamentos experimentais, métodos variados para coleta de dados, incluindo observações diretas do comportamento dos participantes. Destaca-se ainda a importância de estudos com o método FRIENDS (Barrett, 1998, 2012) em outros contextos, como escola e CRAS para a ampliação dos estudos de eficácia deste programa para o contexto brasileiro.

Além dos resultados obtidos por meio do instrumento, que apontam a diminuição do total de sintomas de ansiedade após a participação das crianças no método FRIENDS (Barrett, 1998, 2012), foi possível que os facilitadores observassem, no decorrer do método, o nível de satisfação dos pais e das crianças por meio de feedbacks verbais. Esses contatos indicaram alto nível de interesse e satisfação com o programa, o que pode indicar um critério importante de aceitação e validade ecológica do método FRIENDS (Barrett, 1998, 2012) para o contexto brasileiro.

\section{Referências}

Ahlen, J., Breitholtz, E., Barrett, P. M., \& Gallegos, J. (2012). School-based prevention of anxiety and depression: A pilot study in Sweden. Advances in School Mental Health Promotion, 5(4), 246-257. doi: 10.1080/1754730X.2012.730352

American Psychiatric Association. (2014). DSM-5: Manual diagnóstico e estatístico de transtornos mentais. 5a ed. Porto Alegre: Artmed.

Anticich, S. A. J., Barrett, P. M., Silverman, W., Lacherez, P., \& Gillies, R. (2013). The prevention of childhood anxiety and promotion of resilience among preschool-aged children: a universal school based trial. Advances in School Mental Health Promotion, 6, 93-121. doi: 10.1080. https://doi.org/1 0.1080/1754730X.2013.784616

Asbahr, F. R. (2004). Transtornos ansiosos na infância e adolescência: Aspectos clínicos e neurobiológicos. Jornal de Pediatria, 80(2), 28-34. doi: 10.1590/ S0021-75572004000300005

Barrett, P. M. (1998). Evaluation of cognitive-behavioral group treatments for childhood anxiety disorders. Journal of Clinical Child Psychology. 27, 459-468. doi: 10.1207/s15374424jccp2704_10

Barrett, P. M. (2012). Amigos divertidos: Guia do facilitador para construção de resiliência em crianças de 4 a 7 anos através do brincar. 3a ed. Austrália: Barrett Research Resources PTy Ltd.

Barrett, P. M., Farrell, L. J., Ollendick, T. H., Dadds, M. (2006). Long-term outcomes of an Australian universal prevention trial of anxiety and depression symptoms in children and youth: An svaluation of the Friends Program. Journal of Clinical Child and Adolescent Psychology. 35(3), 403-411. doi: 10.1207/s15374424jccp3503_5.

Brasil, Ministério da Saúde (2015). Saúde Mental em Dados - 12. 10(12) Brasília, DF. Recuperado de http://portalarquivos.saude.gov.br/images/ pdf/2015/outubro/20/12-edicao-do-Saude-Mental-em-Dados.pdf

Buss, P. M. (2009). Uma introdução ao conceito de promoção da saúde. Em Czeresnia, Dina \& C. M. Freitas (Eds.), Promoção da Saúde. (pp. 19-42). Rio de Janeiro: Editora Fiocruz.

DeSousa, D. A., Pereira, A. S., Petersen, C. S., Manfro, G. G., Salum, G. A., \& Koller, S. H. (2014). Psychometric properties of the brazilian-portuguese version of the Spence children's anxiety acale (SCAS): Self- and parent-report versions. Journal of Anxiety Disorders, 28, 427- 436. doi: 10.1016/j. janxdis.2014.03.006

Espírito-Santo, H., \& Daniel, F. (2015). Calcular e apresentar tamanhos do efeito em trabalhos científicos (1): As limitações do $p<0,05$ na análise de diferenças de médias de dois grupos. Revista Portuguesa de Investigação Comportamental e Social, 1(1): 3-16.

Fernandes, L. F. B., Carvalho, F. A., Izbicki, S., \& Melo, M. H. S. (2014). Prevenção Universal de ansiedade Psico-USF, Bragança Paulista, v. 25, n. 3, p. 519-531, jul./set. 2020 
na infância e adolescência: Uma revisão sistemática. Revista Psicologia: Teoria e Prática, 16(3), 83-99. doi: 10.15348/1980-6906/psicologia.v16n3p83-99

Figueredo, L. Z. P. (No prelo). Uma luz no fim do túnel? Programas de prevenção e promoção de saúde mental e a implementação do Método FRIENDS no Brasil.

Friedberg, R. D.; Mcclure, J. M. (2004). A prática de clínica de terapia cognitiva com crianças e adolescentes. Porto Alegre: Artmed.

Gillham, J. E., Shatte, A. J., Reivich, K. (2001). Needed for prevention research: Long-term follow-up and the evaluation of mediators, moderators, and lay providers. Prevention and Treatment.

Graeff-Martins, A. S., \& Fleitlick-Bilyk, B. (2016). Instrumentos de avaliação de uso na infância e adolescência. Em C. Gorenstein, Y. P. Wang \& I. Hungerbuhler (Eds.), Instrumentos de avaliação em saúde mental. Porto Alegre: Artmed.

Iizuka, C., \& Barrett, P. (2011). Programa Friends para tratamento e prevenção de transtornos de ansiedade em crianças e adolescentes. Em C. S. Petersen et al., (Eds.), Terapias cognitivo comportamentais para crianças e adolescentes: Ciência e arte (pp. 264-281). Porto Alegre: Artmed.

Iizuka, C., Barrett, P. M., Gillies, R., Cook, C. R., Miller, D. (2014). The FRIENDS emocional health program for minority groups at risk. J School Health, 84(2), 124-32. doi: 10.1111/josh.12127

Kosters, M. P., Chinapaw, M. J., Zwaanswijk,M., Vander Wal, M. F., Mwjutens, E., \& Koot, H. M. (2012). Study design of 'FRIENDS for life': Process and effect evaluation of an indicated school-based prevention programme for childhood anxiety and depression. Bmc public health, 12(1), 86. doi: 10.1186/1471-2458-12-86.

Liddle, I., \& Macmillan. S. (2010) Evaluating the FRIENDS programme in a Scottish setting. Educational Psychology in Practice, 26(1). doi: 10.1080/02667360903522785

Lock, S., \& Barrett, P. M. (2003). A longitudinal study of developmental differences in universal preventive intervention for child anxiety. Behaviour Change, 20(4), 183-199.

Lohr, S. S., Melo, M. H. S., De Salvo, C. G., \& Silvares, E. F. M. (2013). Prevenção e promoção da
Saúde: Um desafio na formação de psicólogos. Revista de psicologia da Criança e do Adolescente, 4(2), 205-222. Recuperado de http:// revistas.lis.ulusiada.pt/index.php/rpca/article/ viewFile/433/411

Murta, S. G., Gunther, I. A., \& Guzzo, R. S. (2015). Prevenção e promoção em saúde mental no curso de vida: Indicadores para a ação. Em S. G. Murta, C. Leandro-Grança, K. B. Santos, L. Polejack (Eds.), Prevenção e promoção em saúde mental: Fundamentos, planejamento e estratégias de prevenção (pp. 75-93). Novo Hamburgo: Sinopsys.

Neil, A. L., \& Christensen, H. (2009). Efficacy and effectiveness of school-based prevention and early intervention programs for anxiety. Clinical Psychology Review, 29(3), 208-215. doi: 10.1016/j. cpr.2009.01.002.

NREPP. (2012). National Registration of evidencebased programs and pratices. Recuperado de https://nrepp.samhsa.gov/legacy/viewintervention.aspx?id $=334$

Organization, W. H. (2012). Risks to Mental Health: An overview of vulnerabilities and risk factors. Recuperado de http://www.who.int/mental_health/ mhgap/risks_to_mental_health_EN_27_08_12. pdf

Organization., W. H. (2016). Investing in treatment for depression and anxiety leads to fourfold return. Recuperado de http://www. who.int/mediacentre/news/releases/2016/ depression-anxiety-treatment/en/

Pavoski, G. T. T., Toni, C. G. S., Batista, A. P., \& Ignachewski, C. L. (2018). Prevenção universal e promoção de saúde em grupo de crianças a partir do Método FRIENDS. Psico (Porto Alegre), 49(2), 148-158. doi: 10.15448/1980-8623.2018.2.26501

Pahl, K. M., \& Barrett, P. M. (2010). Preventing anxiety and promoting social and emotional strength in preschool children: a universal evaluation of the Fun FRIENDS program. Advances in School Mental Health Promotion, 3(3), 14-25.

Pine D. S., Cohen P., Gurley D., Brook J., Ma Y. (1998). The risk for early adulthood anxiety and depressive disorders in adolescents with anxiety and depressive disorders. Archives of General Psychiatry.55:56-64 
Spence, S. H. (1999). Spence Children's Anxiety Scale (parent version). Brisbane: University of Queensland.

Stallard, P. (2002). Ansiedade: Terapia cognitivo-comportamental para crianças e jovens. Porto Alegre: Artmed.

Stallard, P. (2010). Mental health prevention in UK classrooms: The FRIENDS anxiety prevention programme. Emotional and Behavioural Difficulties, 15(1), 23-35. doi: 10.1080/13632750903512381

Stallard, P., Simpson, N., Anderson, S., Carter, T., Osborn, C., \& Bush, S. (2005). An evaluation of the FRIENDS programme: A cognitive behaviour therapy intervention to promote emotional resilience. Arch Dis Child, 90,1016-1019. doi: 10.1136/ adc.2004.068163

Teste de Wilcoxon. (1945). Disponível em http://www. inf.ufsc.br/ $\sim$ vera.carmo/Testes_de_Hi\%20poteses/Teste_nao_parametrico_Wilcoxon.pdf

Tortella-Feliu, M., Servera, M., Balle, M., \& Fullana, M. A. (2004). Viabilidad de um programa de prevención selectiva de los problemas de ansiedade em la infância aplicado em la escuela. International Journal of Clinical and Health Psychology, 4(2),
371-38. Recuperado de http://www.redalyc.org/ articulo.oa?id $=33740209$

Vianna, R. R. A. B., Campos, A. A., Landeira-Fernandez, J. (2009). Transtornos de ansiedade na infância e adolescência: uma revisão. Rev. bras. ter. cogn. 5(1), 46-61. Recuperado em 27 de maio de 2018, de http://pepsic.bvsalud.org/scielo.php?script $=$ sci_ arttext\&pid $=$ S1808-56872009000100005\&lng $=$ pt $\& \operatorname{lng}=\mathrm{pt}$

Weems, C. F., \& Stickle, T. R. (2005). Anxiety disorders in childhood: Casting a nomological net. Clinical Child \& Family Psychology Review. 8(2), 107-34. doi: 10.1007/s10567-005-4751-2

Zeggio, L., \& Araújo, L. B. T. S. (2015). Desenvolvimento de habilidades socioemocionais na escola e a experiência bem sucedida do Método FRIENDS: Quando as neurociências e a educação se encontram pelo caminho da psicologia positiva. Em M. Barr (Ed.), Neurociências e educação na primeira infância: Progressos e obstáculos. Brasília: Senado Federal, Comissão de valorização da primeira infância e cultura da paz.

Recebido em: 21/10/2017

Reformulado em: 12/03/2019 Aprovado em: 09/09/2019 
Sobre as autoras:

Letícia Guancino é psicóloga graduada pela Universidade Estadual do Centro-Oeste-PR.

ORCID: https://orcid.org/0000-0002-4202-4081

E-mail: leticiaaguancino@gmail.com

Caroline Guisantes de Salvo Toni é psicóloga graduada pela Universidade Federal do Paraná, doutora em Psicologia Clínica pela Universidade de São Paulo e professora adjunta do Curso de Psicologia da Universidade Estadual do Centro-Oeste-PR.

ORCID: https://orcid.org/0000-0003-2114-1964

E-mail: carolineguisantes@yahoo.com.br

Ana Priscila Batista é psicóloga graduada pela Universidade Estadual de Londrina (UEL), especialista em Terapia Comportamental e Cognitiva: Teoria e prática pela Universidade de São Paulo (USP), mestre em Psicologia Experimental pela Universidade de São Paulo (USP), doutora em Educação pela Universidade Federal do Paraná (UFPR) e professora adjunta do Curso de Psicologia da Universidade Estadual do Centro-Oeste-PR.

ORCID: https://orcid.org/0000-0001-9849-3998

E-mail: anapribatista@yahoo.com.br

Contato com as autoras:

Universidade Estadual do Centro-Oeste

Rua Professora Maria Roza Zanon de Almeida, S/N, Engenheiro Gutierrez

Irati-PR, Brasil

CEP: 84505-677 